\title{
The integral representation of the solution of some mixed problem for evolutionary equations
}

\author{
Ivan Konet and Tetiana Pylypiuk
}




\title{
THE INTEGRAL REPRESENTATION OF THE SOLUTION OF SOME MIXED PROBLEM FOR EVOLUTIONARY EQUATIONS
}

\author{
I. KONET AND T. PYLYPIUK
}

Received 14 October, 2013

\begin{abstract}
By means of the method of hybrid integral transform of Euler-Fourier-Bessel type with spectral parameter the integral representation of exact analytical solution of mixed problem for the system of equations of parabolic type in the three-part segment $[0, R]$ is obtained under the assumption that the limits are soft. Modeling of evolutionary processes is performed by the method of hybrid differential Euler-Fourier-Bessel operators.
\end{abstract}

2010 Mathematics Subject Classification: 34D20

Keywords: parabolic equation, initial and boundary conditions, conjugate conditions, the differential operator, hybrid integral transform, Green functions, the influence functions

\section{INTRODUCTION}

The theory of boundary value problems for partial differential equations is an important section of the modern theory of differential equations which is intensively developing in the present time. The popularity of the problem is the consequence of the significance of its results in the development of many mathematical problems, as well as of its numerous applications in mathematical modeling of different processes and phenomenon of physics, chemistry, mechanics, biology, medicine, economics, engineering.

It is well known that the complexity of a boundary-value problem significantly depends on the coefficients of equations (different types of degeneracy and features) and the geometry of domain (smoothness of the boundary, the presence of corner points, etc.) in which the problem is considered. The dependence of the properties of solutions of boundary value problems for linear, quasi-linear, and certain classes of nonlinear equations in homogeneous domains on the above-mentioned properties of the coefficients of equations and geometry of domain are studied in detail, and functional spaces of correctness of problems for some domains are constructed [2, 13].

However, many important applied problems of thermophysics, thermodynamics, theory of elasticity, theory of electrical circuits, theory of vibrations lead to boundary value problems for partial differential equations not only in homogeneous domains 
(when the coefficients of the equations are continuous), but also in inhomogeneous and piecewise homogeneous domains if the coefficients of the equations are piecewise continuous or piecewise constant $[1,14]$.

Besides the method of separation of variables [17], which is one of the important and effective methods of studying linear boundary value problems for partial differential equations, there is the method of integral transforms, which makes it possible to obtain analytical solutions of some boundary-value problems via their integral images. It is also worth mentioning that for a rather wide class of problems (in piecewise homogeneous domains) there exists the effective method of hybrid integral transforms. They are generated by differential operators, when on each of the components of the piecewise homogeneous domain one considers different differential operators or differential operators of the same form, but with different sets of coefficients $[3-5,7]$.

In theoretical investigations and in applied problems the most frequently used differential operators are of second order, in particular the Fourier differential operator [16]

$$
F=\frac{d^{2}}{d r^{2}}
$$

the Euler differential operator [9]

$$
B_{\alpha}^{*}=r^{2} \frac{d^{2}}{d r^{2}}+(2 \alpha+1) r \frac{d}{d r}+\alpha^{2},
$$

the Bessel differential operator [11]

$$
B_{v, \alpha}=\frac{d^{2}}{d r^{2}}+\frac{2 \alpha+1}{r} \frac{d}{d r}-\frac{v^{2}-\alpha^{2}}{r^{2}},
$$

the generalized Legendre differential operator [6]

$$
\Lambda_{(\mu)}=\frac{d^{2}}{d r^{2}}+c t h r \frac{d}{d r}+\frac{1}{4}+\frac{1}{2}\left(\frac{\mu_{1}^{2}}{1-c h r}+\frac{\mu_{2}^{2}}{1+c h r}\right), \mu=\left(\mu_{1}, \mu_{2}\right)
$$

and Kontorovich-Lebedev differential operator [10]

$$
B_{\alpha}=r^{2} \frac{d^{2}}{d r^{2}}+(2 \alpha+1) r \frac{d}{d r}+\alpha^{2}-\lambda^{2} r^{2}
$$

If $\theta(x)$ is the Heaviside step function [15] and $L_{j}$ is one of listed differential operators, then we can always create hybrid differential operator that corresponds to the geometric structure of piecewise homogeneous domain.

For example, for the piecewise homogeneous interval $\left(R_{0}, R_{1}\right) \cup\left(R_{1}, R_{2}\right) \cup\left(R_{2}, R\right)$ it is possible to create hybrid differential operator (HDO)

$$
\begin{gathered}
M=\theta\left(r-R_{0}\right) \theta\left(R_{1}-r\right) a_{1}^{2} L_{1}+\theta\left(r-R_{1}\right) \theta\left(R_{2}-r\right) a_{2}^{2} L_{2}+ \\
+\theta\left(r-R_{2}\right) \theta(R-r) a_{3}^{2} L_{3} ; a_{j}^{2}=\text { const } .
\end{gathered}
$$


It is obvious that operator $L_{1}$ is defined in the interval $\left(R_{0}, R_{1}\right)$, operator $L_{2}$ is defined in the interval $\left(R_{1}, R_{2}\right)$, and operator $L_{3}$ is defined in the interval $\left(R_{2}, R\right)$. It is clear, that if we change the order of operators $L_{1}, L_{2}, L_{3}$ we get other hybrid differential operator.

\section{FORMULATION OF THE PROBLEM}

Here we consider the problem of the structure of the solution which is bounded in the set $D_{2}=\left\{(t, r): t \in(0 ;+\infty) ; r \in I_{2}=\left(0, R_{1}\right) \cup\left(R_{1}, R_{2}\right) \cup\left(R_{2}, R\right) ; R<+\infty\right\}$ for the system of evolutionary partial differential equations of parabolic type [17]

$$
\begin{gathered}
\frac{\partial u_{1}}{\partial t}+\gamma_{1}^{2} u_{1}(t, r)-a_{1}^{2} B_{\alpha_{1}}^{*}\left[u_{1}(t, r)\right]=f_{1}(t, r), r \in\left(0, R_{1}\right), \\
\frac{\partial u_{2}}{\partial t}+\gamma_{2}^{2} u_{2}(t, r)-a_{2}^{2} F\left[u_{2}(t, r)\right]=f_{2}(t, r), r \in\left(R_{1}, R_{2}\right), \\
\frac{\partial u_{3}}{\partial t}+\gamma_{3}^{2} u_{3}(t, r)-a_{3}^{2} B_{v, \alpha_{2}}\left[u_{3}(t, r)\right]=f_{3}(t, r), r \in\left(R_{2}, R\right)
\end{gathered}
$$

with zero initial conditions, boundary conditions

$$
\lim _{r \rightarrow 0} \frac{\partial^{k} u_{1}(t, r)}{\partial r^{k}}=0,\left.\quad L_{22}^{3}\left[u_{3}(t, r)\right]\right|_{r=R}=g_{R}(t)
$$

and conjugate conditions

$$
\left.\left(L_{j 1}^{k}\left[u_{k}(t, r)\right]-L_{j 2}^{k}\left[u_{k+1}(t, r)\right]\right)\right|_{r=R_{k}}=\omega_{j k}(t) ; j, k=1,2 .
$$

Here $B_{\alpha_{1}}^{*}$ is Euler differential operator (1.2) with parameter $\alpha=\alpha_{1}, F$ is Fourier differential operator (1.1), $B_{v, \alpha_{2}}$ is Bessel differential operator (1.3) with parameter $\alpha=\alpha_{2} ; 2 \alpha_{j}+1>0, v \geq \alpha_{2}$.

In the boundary conditions (2.2) at the point $r=R$ and in the conjugate conditions (2.3) the differential operators

$$
L_{j k}^{m}=\left(\alpha_{j k}^{m}+\delta_{j k}^{m} \frac{\partial}{\partial t}\right) \frac{\partial}{\partial r}+\beta_{j k}^{m}+\gamma_{j k}^{m} \frac{\partial}{\partial t} ; j, k=1,2 ; m=1,2,3,
$$

take part.

We assume that the following conditions on the coefficients are true: $\alpha_{j k}^{m} \geq 0$, $\beta_{j k}^{m} \geq 0, \delta_{j k}^{m} \geq 0, \gamma_{j k}^{m} \geq 0, a_{j}>0, c_{j 1, k}=\alpha_{2 j}^{k} \beta_{1 j}^{k}-\alpha_{1 j}^{k} \beta_{2 j}^{k}, c_{11, k} \cdot c_{21, k}>0 ;$ $\alpha_{22}^{3}+\beta_{22}^{3} \neq 0, c_{j 2, k}=\delta_{2 j}^{k} \gamma_{1 j}^{k}-\delta_{1 j}^{k} \gamma_{2 j}^{k}=0, \alpha_{11}^{k} \gamma_{21}^{k}-\alpha_{21}^{k} \gamma_{11}^{k}=\beta_{11}^{k} \delta_{21}^{k}-\beta_{21}^{k} \delta_{11}^{k}$, $\alpha_{12}^{k} \gamma_{22}^{k}-\alpha_{22}^{k} \gamma_{12}^{k}=\beta_{12}^{k} \delta_{22}^{k}-\beta_{22}^{k} \delta_{12}^{k}, j, k=1,2$.

Remark 1. The presence of the operator $\frac{\partial}{\partial t}$ in the boundary conditions at the point $r=R$ and in the conjugate conditions can be interpreted as the softness of the boundary of the domain when it reflects heat waves. 
Remark 2. In the case of hard boundary of the domain for the reflection of waves $\left(\delta_{j k}^{m}=0, \gamma_{j k}^{m}=0\right)$, we have mixed a problem with classical boundary conditions and classical conjugate conditions. Its solution can be derived from the solution of the problem (2.1)-(2.3) as a particular case.

\section{HYBRID EULER-FOURIER-BESSEL INTEGRAL TRANSFORM WITH SPECTRAL} PARAMETER

Let's consider in the set $I_{2}$ the hybrid differential operator

$$
M_{v,(\alpha)}=\theta(r) \theta\left(R_{1}-r\right) B_{\alpha_{1}}^{*}+\theta\left(r-R_{1}\right) \theta\left(R_{2}-r\right) F+\theta\left(r-R_{2}\right) \theta(R-r) B_{v, \alpha_{2}} .
$$

Here $\theta(x)$ is the Heaviside step function, $(\alpha)=\left(\alpha_{1}, \alpha_{2}\right)$.

Definition 1. The domain of definition of the HDO $M_{\nu,(\alpha)}$ is the set $G$ of functions $g(r)=\left\{g_{1}(r) ; g_{2}(r) ; g_{3}(r)\right\}$ with the following properties:

1) the function $f(r)=\left\{B_{\alpha_{1}}^{*}\left[g_{1}(r)\right] ; g_{2}^{\prime \prime}(r) ; B_{v, \alpha_{2}}\left[g_{3}(r)\right]\right\}$ is continuous in the set $I_{2}$;

2) functions $g_{j}(r)$ satisfy the boundary conditions

$$
\lim _{r \rightarrow 0} \frac{d^{k} u_{1}(r)}{d r^{k}}=0 ; k=0,1 ;\left.\quad\left(\tilde{\alpha}_{22}^{3} \frac{d}{d r}+\tilde{\beta}_{22}^{3}\right) g_{3}(r)\right|_{r=R}=0
$$

and conjugate conditions

$$
\left.\left[\left(\tilde{\alpha}_{j 1}^{k} \frac{d}{d r}+\tilde{\beta}_{j 1}^{k}\right) g_{k}(r)-\left(\tilde{\alpha}_{j 2}^{k} \frac{d}{d r}+\tilde{\beta}_{j 2}^{k}\right) g_{k+1}(r)\right]\right|_{r=R_{k}}=0 ; j, k=1,2 .
$$

Here $\tilde{\alpha}_{j m}^{k}=\alpha_{j m}^{k}-\left(\beta^{2}+\gamma^{2}\right) \delta_{j m}^{k}, \tilde{\beta}_{j m}^{k}=\beta_{j m}^{k}-\left(\beta^{2}+\gamma^{2}\right) \gamma_{j m}^{k}, \beta \in(0,+\infty)$ is the spectral parameter, $\gamma^{2}=\max \left\{\gamma_{1}^{2} ; \gamma_{2}^{2} ; \gamma_{3}^{2}\right\}$.

From the conjugate conditions (3.3) by direct calculations we obtain the basic identity for the two functions $u(r)=\left\{u_{1}(r) ; u_{2}(r) ; u_{3}(r)\right\} \in G$ and $v(r)=\left\{v_{1}(r) ; v_{2}(r) ; v_{3}(r)\right\} \in G$ :

$$
\begin{gathered}
{\left.\left[u_{k}^{\prime}(r) v_{k}(r)-u_{k}(r) v_{k}^{\prime}(r)\right]\right|_{r=R_{k}}=} \\
=\left.\frac{c_{21, k}}{c_{11, k}}\left[u_{k+1}^{\prime}(r) v_{k+1}(r)-u_{k+1}(r) v_{k+1}^{\prime}(r)\right]\right|_{r=R_{k}} ; k=1,2 .
\end{gathered}
$$

Let's define the values

$$
a_{1}^{2} \sigma_{1}=1, \quad a_{2}^{2} \sigma_{2}=\frac{c_{21,1}}{c_{11,1}} R_{1}^{2 \alpha_{1}+1}, \quad a_{3}^{2} \sigma_{3}=\frac{c_{21,1} c_{21,2}}{c_{11,1} c_{11,2}} \frac{R_{1}^{2 \alpha_{1}+1}}{R_{2}^{2 \alpha_{2}+1}},
$$

the weight function

$$
\begin{aligned}
\sigma(r)= & \theta(r) \theta\left(R_{1}-r\right) \sigma_{1} r^{2 \alpha_{1}-1}+\theta\left(r-R_{1}\right) \theta\left(R_{2}-r\right) \sigma_{2} \\
& +\theta\left(r-R_{2}\right) \theta(R-r) \sigma_{3} r^{2 \alpha_{2}+1}
\end{aligned}
$$


and the scalar product

$$
\begin{gathered}
(u(r), v(r))=\int_{0}^{R} u(r) v(r) \sigma(r) d r \equiv \int_{0}^{R_{1}} u_{1}(r) v_{1}(r) \sigma_{1} r^{2 \alpha_{1}-1} d r+ \\
+\int_{R_{1}}^{R_{2}} u_{2}(r) v_{2}(r) \sigma_{2} d r+\int_{R_{2}}^{R} u_{3}(r) v_{3}(r) \sigma_{3} r^{2 \alpha_{2}+1} d r .
\end{gathered}
$$

We now check, that HDO $M_{v,(\alpha)}$ is a self-conjugate operator, namely

$$
\left(M_{\nu,(\alpha)}[u], v\right)=\left(u, M_{v,(\alpha)}[v]\right) .
$$

According to the equality (3.6) we have, that

$$
\begin{gathered}
\left(M_{v,(\alpha)}[u], v(r)\right)=\int_{0}^{R_{1}}\left(a_{1}^{2} B_{\alpha_{1}}^{*}\left[u_{1}\right]\right) v_{1} \sigma_{1} r^{2 \alpha_{1}-1} d r+ \\
+\int_{R_{1}}^{R_{2}}\left(a_{2}^{2} \frac{d^{2} u_{2}}{d r^{2}}\right) v_{2}(r) \sigma_{2} d r+\int_{R_{2}}^{R}\left(a_{3}^{2} B_{v, \alpha_{2}}\left[u_{3}(r)\right] v_{3}(r) \sigma_{3} r^{2 \alpha_{2}+1} d r .\right.
\end{gathered}
$$

Let's integrate in (3.8) by parts twice. We get:

$$
\begin{aligned}
& \left(M_{v,(\alpha)}[u], v\right)=\left.a_{1}^{2} \sigma_{1}\left[r^{2 \alpha_{1}+1}\left(\frac{d u_{1}}{d r} v_{1}-u_{1} \frac{d v_{1}}{d r}\right)\right]\right|_{0} ^{R_{1}}+\int_{0}^{R_{1}} u_{1}(r)\left(a_{1}^{2} B_{\alpha_{1}}^{*}\left[v_{1}\right]\right) \times \\
& \times \sigma_{1} r^{2 \alpha_{1}-1} d r+\left.a_{2}^{2} \sigma_{2}\left(\frac{d u_{2}}{d r} v_{2}-u_{2} \frac{d v_{2}}{d r}\right)\right|_{R_{1}} ^{R_{2}}+\int_{R_{1}}^{R_{2}} u_{2}(r)\left(a_{2}^{2} \frac{d^{2} v_{2}}{d r^{2}}\right) \sigma_{2} d r+\quad(3.9) \\
& +\left.a_{3}^{2} \sigma_{3}\left[r^{2 \alpha_{2}+1}\left(\frac{d u_{3}}{d r} v_{3}-u_{3} \frac{d v_{3}}{d r}\right)\right]\right|_{R_{2}} ^{R}+\int_{R_{2}}^{R} u_{3}(r)\left(a_{3}^{2} B_{v, \alpha_{2}}\left[v_{3}\right]\right) \sigma_{3} r^{2 \alpha_{2}+1} d r
\end{aligned}
$$

Due to the first condition of (3.2), the term outside the integral at the point $r=0$ is equal to zero.

At the point $r=R_{1}$, because of the basic identity (3.4) when $k=1$ we have

$$
\begin{gathered}
\left.a_{1}^{2} \sigma_{1} R_{1}^{2 \alpha_{1}+1}\left(u_{1}^{\prime} v_{1}-u_{1} v_{1}^{\prime}\right)\right|_{r=R_{1}}-\left.a_{2}^{2} \sigma_{2}\left(u_{2}^{\prime} v_{2}-u_{2} v_{2}^{\prime}\right)\right|_{r=R_{1}}= \\
=\left.\left(a_{1}^{2} \sigma_{1} R_{1}^{2 \alpha_{1}+1} \frac{c_{21,1}}{c_{11,1}}-a_{2}^{2} \sigma_{2}\right)\left(u_{2}^{\prime} v_{2}-u_{2} v_{2}^{\prime}\right)\right|_{r=R_{1}}=
\end{gathered}
$$




$$
=\left.\left(\frac{c_{21,1}}{c_{11,1}} R_{1}^{2 \alpha_{1}+1}-\frac{c_{21,1}}{c_{11,1}} R_{1}^{2 \alpha_{1}+1}\right)\left(u_{2}^{\prime} v_{2}-u_{2} v_{2}^{\prime}\right)\right|_{r=R_{1}}=0 .
$$

At the point $r=R_{2}$, because of the basic identity (3.4) when $k=2$ we have

$$
\begin{gathered}
\left.a_{2}^{2} \sigma_{2}\left(u_{2}^{\prime} v_{2}-u_{2} v_{2}^{\prime}\right)\right|_{r=R_{2}}-\left.a_{3}^{2} \sigma_{3} R_{2}^{2 \alpha_{2}+1}\left(u_{3}^{\prime} v_{3}-u_{3} v_{3}^{\prime}\right)\right|_{r=R_{2}}= \\
=\left.\left(a_{2}^{2} \sigma_{2} \frac{c_{21,2}}{c_{11,2}}-a_{3}^{2} \sigma_{3} R_{2}^{2 \alpha_{2}+1}\right)\left(u_{3}^{\prime} v_{3}-u_{3} v_{3}^{\prime}\right)\right|_{r=R_{2}}= \\
=\left(\frac{c_{21,1}}{c_{11,1}} \frac{c_{21,2}}{c_{11,2}} R_{1}^{2 \alpha_{1}+1}-\left.\frac{c_{21,1} c_{21,2}}{c_{11,1} c_{11,2}} \frac{R_{1}^{2 \alpha_{1}+1}}{R_{2}^{2 \alpha_{2}+1}} R_{2}^{2 \alpha_{2}+1}\left(u_{3}^{\prime} v_{3}-u_{3} v_{3}^{\prime}\right)\right|_{r=R_{2}}=\right. \\
=\left.\frac{c_{21,1} c_{21,2}}{c_{11,1} c_{11,2}} R_{1}^{2 \alpha_{1}+1}(1-1)\left(u_{3}^{\prime} v_{3}-u_{3} v_{3}^{\prime}\right)\right|_{r=R_{2}} \equiv 0 .
\end{gathered}
$$

If $\tilde{\alpha}_{22}^{3} \neq 0$, then expression

$$
\begin{gathered}
\left.\left(\frac{d u_{3}}{d r} v_{3}-u_{3} \frac{d v_{3}}{d r}\right)\right|_{r=R}=\left(\tilde{\alpha}_{22}^{3}\right)^{-1}\left[\left.\left(\tilde{\alpha}_{22}^{3} \frac{d u_{3}}{d r}+\tilde{\beta}_{22}^{3} u_{3}\right)\right|_{r=R} \cdot v_{3}(R)-\left(\tilde{\alpha}_{22}^{3} \frac{d v_{3}}{d r}+\right.\right. \\
\left.\left.+\tilde{\beta}_{22}^{3} v_{3}\right)\left.\right|_{r=R} \cdot u_{3}(R)\right]=\left(\tilde{\alpha}_{22}^{3}\right)^{-1}\left(0 \cdot v_{3}(R)-0 \cdot u_{3}(R)\right)=0 .
\end{gathered}
$$

Therefore, in the equation (3.9) the terms outside the integral are equal to zero and we have that

$$
\left(M_{\nu,(\alpha)}[u], v\right)=\left(u, M_{v,(\alpha)}[v]\right) .
$$

So HDO $M_{v,(\alpha)}$ is self-conjugate. Hence its eigenvalues form a real spectrum. Since the HDO $M_{v,(\alpha)}$ has one singular point $r=0$, then it's spectrum is continuous [9]. We can assume, that spectral parameter $\beta \in(0,+\infty)$. Real spectral function

$$
V_{v,(\alpha)}(r, \beta)=\sum_{k=1}^{3} \theta\left(r-R_{k-1}\right) \theta\left(R_{k}-r\right) V_{\nu,(\alpha) ; k}(r, \beta), \quad R_{0}=0, R_{3}=R,
$$

corresponds to it.

Herewith functions $V_{v,(\alpha) ; k}(r, \beta)$ must satisfy respectively the differential equations

$$
\begin{gathered}
\left(B_{\alpha_{1}}^{*}+b_{1}^{2}\right) V_{v,(\alpha) ; 1}(r, \beta)=0, r \in\left(0, R_{1}\right), \\
\left(F+b_{2}^{2}\right) V_{\nu,(\alpha) ; 2}(r, \beta)=0, r \in\left(R_{1}, R_{2}\right), \\
\left(B_{v, \alpha_{2}}+b_{3}^{2}\right) V_{v,(\alpha) ; 3}(r, \beta)=0, r \in\left(R_{2}, R\right),
\end{gathered}
$$

boundary conditions (3.2) and conjugate conditions (3.3); $b_{j}^{2}=a_{j}^{-2}\left(\beta^{2}+k_{j}^{2}\right), k_{j}^{2} \geq$ $0, j=\overline{1,3}$.

The fundamental system of solutions for Euler differential equation $\left(B_{\alpha_{1}}^{*}+b_{1}^{2}\right) v=$ 0 is formed by functions $r^{-\alpha_{1}} \cos \left(b_{1} \ln r\right)$ and $r^{-\alpha_{1}} \sin \left(b_{1} \ln r\right)$ [16]; the fundamental system of solutions for Fourier differential equation $\left(F+b_{2}^{2}\right) v=0$ is formed by 
functions $\cos b_{2} r$ and $\sin b_{2} r$ [16]; the fundamental system of solutions for Bessel differential equation $\left(B_{v, \alpha_{2}}+b_{3}^{2}\right) v=0$ is formed by functions $J_{v, \alpha_{2}}\left(b_{3} r\right)$ and $N_{v, \alpha_{2}}\left(b_{3} r\right)$ [11].

The presence of the fundamental system of solutions allows us to put

$$
\begin{gathered}
V_{v,(\alpha) ; 1}(r, \beta)=A_{1} r^{-\alpha_{1}} \cos \left(b_{1} \ln r\right)+B_{1} r^{-\alpha_{1}} \sin \left(b_{1} \ln r\right), \\
V_{v,(\alpha) ; 2}(r, \beta)=A_{2} \cos \left(b_{2} r\right)+B_{2} \sin \left(b_{2} r\right), \\
V_{v,(\alpha) ; 3}(r, \beta)=A_{3} J_{v, \alpha_{2}}\left(b_{3} r\right)+B_{3} N_{v, \alpha_{2}}\left(b_{3} r\right) .
\end{gathered}
$$

Conjugate conditions (3.3) and boundary condition at the point $r=R$ for the determination of the six variables $A_{j}, B_{j}(j=\overline{1,3})$ give homogeneous algebraic system of five equations:

$$
\begin{gathered}
Y_{\alpha_{1} ; j 1}^{11}\left(b_{1}, R_{1}\right) A_{1}+Y_{\alpha_{1} ; j 1}^{12}\left(b_{1}, R_{1}\right) B_{1}-v_{j 2}^{11}\left(b_{2} R_{1}\right) A_{2}-v_{j 2}^{12}\left(b_{2} R_{1}\right) B_{2}=0, j=1,2 ; \\
v_{j 1}^{21}\left(b_{2} R_{2}\right) A_{2}+v_{j 1}^{22}\left(b_{2} R_{2}\right) B_{2}-u_{v, \alpha_{2} ; j 2}^{21}\left(b_{3} R_{2}\right) A_{3}-u_{v, \alpha_{2} ; j 2}^{22}\left(b_{3} R_{2}\right) B_{3}=0 ; \\
u_{v, \alpha_{2} ; 22}^{31}\left(b_{3} R\right) A_{3}+u_{v, \alpha_{2} ; 22}^{32}\left(b_{3} R\right) B_{3}=0 .
\end{gathered}
$$

Algebraic system (3.13)) is compatible. It's solution is constructed in standard way.

Let's suppose, that $A_{3}=-A_{0} u_{v, \alpha_{2} ; 22}^{32}\left(b_{3}, R\right), B_{3}=A_{0} u_{v, \alpha_{2} ; 22}^{31}\left(b_{3}, R\right)$, here $A_{0}$ is subjected to be defined. At such choice of $A_{3}, B_{3}$ the last equation of the system becomes the identity. For determination of the values $A_{2}, B_{2}$ we obtain the algebraic system:

$$
\begin{aligned}
& v_{j 1}^{21}\left(b_{2} R_{2}\right) A_{2}+v_{j 1}^{22}\left(b_{2} R_{2}\right) B_{2}=-A_{0}\left[u_{v, \alpha_{2} ; j 2}^{21}\left(b_{3} R_{2}\right) u_{v, \alpha_{2} ; 22}^{32}\left(b_{3} R\right)-\right. \\
& \left.-u_{v, \alpha_{2} ; j 2}^{22}\left(b_{3} R_{2}\right) u_{v, \alpha_{2} ; 22}^{31}\left(b_{3} R\right)\right] \equiv-A_{0} \delta_{v, \alpha_{2} ; j 2}\left(b_{3} R_{2}, b_{3} R\right), j=1,2 .
\end{aligned}
$$

Functions

$$
\begin{aligned}
A_{2} & =\frac{A_{0}}{c_{11,2} b_{2}}\left[\delta_{v, \alpha_{2} ; 22}\left(b_{3} R_{2}, b_{3} R\right) v_{11}^{22}\left(b_{2} R_{2}\right)-\delta_{v, \alpha_{2} ; 12}\left(b_{3} R_{2}, b_{3} R\right) v_{21}^{22}\left(b_{2} R_{2}\right)\right], \\
B_{2} & =\frac{A_{0}}{c_{11,2} b_{2}}\left[\delta_{v, \alpha_{2} ; 12}\left(b_{3} R_{2}, b_{3} R\right) v_{21}^{21}\left(b_{2} R_{2}\right)-\delta_{v, \alpha_{2} ; 22}\left(b_{3} R_{2}, b_{3} R\right) v_{11}^{21}\left(b_{2} R_{2}\right)\right]
\end{aligned}
$$

are the solution of the algebraic system (3.14).

Considering $A_{2}, B_{2}$ to be known let's consider the algebraic system on values $A_{1}$, $B_{1}$ :

$$
Y_{\alpha_{1} ; j 1}^{11}\left(b_{1}, R_{1}\right) A_{1}+Y_{\alpha_{1} ; j 1}^{12}\left(b_{1}, R_{1}\right) B_{1}=A_{0} c_{11,2} b_{2} a_{v, \alpha_{2} ; j}(\beta), j=1,2 .
$$

In the system (3.16) we accept the denotation:

$$
\begin{gathered}
\delta_{j k}\left(b_{2} R_{1}, b_{2} R_{2}\right)=v_{j 2}^{11}\left(b_{2} R_{1}\right) v_{k 1}^{22}\left(b_{2} R_{2}\right)-v_{j 2}^{12}\left(b_{2} R_{1}\right) v_{k 1}^{21}\left(b_{2} R_{2}\right), j, k=1,2 ; \\
a_{v, \alpha_{2} ; j}(\beta)=\delta_{v, \alpha_{2} ; 22}\left(b_{3} R_{2}, b_{3} R\right) \delta_{j 1}\left(b_{2} R_{1}, b_{2} R_{2}\right)-
\end{gathered}
$$




$$
-\delta_{v, \alpha_{2} ; 12}\left(b_{3} R_{2}, b_{3} R\right) \delta_{j 2}\left(b_{2} R_{1}, b_{2} R_{2}\right) .
$$

Algebraic system (3.16) has the unique solution [8]:

$$
\begin{gathered}
A_{0}=c_{11,1} b_{1} R_{1}^{-\left(2 \alpha_{1}+1\right)} c_{11,2} b_{2} ; \quad A_{1}=\omega_{\nu,(\alpha) ; 2}(\beta), \quad B_{1}=-\omega_{\nu,(\alpha) ; 1}(\beta) ; \\
\omega_{\nu,(\alpha) ; j}(\beta)=a_{v, \alpha_{2} ; 1}(\beta) Y_{\alpha_{1} ; 21}^{1 j}\left(b_{1}, R_{1}\right)-a_{v, \alpha_{2} ; 2}(\beta) Y_{\alpha_{1} ; 11}^{1 j}\left(b_{1}, R_{1}\right) ; j=1,2 .
\end{gathered}
$$

Substituting values $A_{j}, B_{j}$ to equality (3.12) according to the formulas (3.15) and (3.17), we obtain the functions

$$
\begin{gathered}
V_{v,(\alpha) ; 1}(r, \beta)=\omega_{v,(\alpha) ; 2}(\beta) r^{-\alpha_{1}} \cos \left(b_{1} \ln r\right)-\omega_{v,(\alpha) ; 1}(\beta) r^{-\alpha_{1}} \sin \left(b_{1} \ln r\right), \\
V_{v,(\alpha) ; 2}(r, \beta)=\frac{c_{11,1} b_{1}}{R_{1}^{2 \alpha_{1}+1}}\left[\delta_{v, \alpha_{2} ; 22}\left(b_{3} R_{2}, b_{3} R\right) \varphi_{11}^{2}\left(b_{2} R_{2}, b_{2} r\right)-\right. \\
\left.-\delta_{v, \alpha_{2} ; 12}\left(b_{3} R_{2}, b_{3} R\right) \varphi_{21}^{2}\left(b_{2} R_{2}, b_{2} r\right)\right], \\
\varphi_{j 1}^{2}\left(b_{2} R_{2}, b_{2} r\right)=v_{j 1}^{22}\left(b_{2} R_{2}\right) \cos b_{2} r-v_{j 1}^{21}\left(b_{2} R_{2}\right) \sin b_{2} r \\
V_{v,(\alpha) ; 3}\left(r, \beta_{n}\right)=\frac{c_{11,1} b_{1}}{R_{1}^{2 \alpha_{1}+1}} c_{11,2} b_{2}\left[u_{v, \alpha_{2} ; 22}^{31}\left(b_{3} R\right) N_{v, \alpha_{2}}\left(b_{3} r\right)-\right. \\
\left.-u_{v, \alpha_{2} ; 22}^{32}\left(b_{3} R\right) J_{v, \alpha_{2}}\left(b_{3} r\right)\right] .
\end{gathered}
$$

According to the formula (3.10) spectral function $V_{v,(\alpha)}(r, \beta)$ becomes known (defined).

Let's introduce a spectral density into consideration

$$
\Omega_{v,(\alpha)}(\beta)=\beta\left[b_{1}(\beta)\right]^{-1}\left(\left[\omega_{\nu,(\alpha) ; 1}(\beta)\right]^{2}+\left[\omega_{\nu,(\alpha) ; 2}(\beta)\right]^{2}\right)^{-1} .
$$

The presence of spectral function $V_{v,(\alpha)}(r, \beta)$, weight function $\sigma(r)$ and spectral density $\Omega_{v,(\alpha)}(\beta)$ makes it possible to determine the direct $H_{v,(\alpha)}$ and inverse $H_{v,(\alpha)}^{-1}$ hybrid integral transform (HIT) of Euler - Fourier - Bessel type with the spectral parameter, generated in the set $I_{2}$ by HDO $M_{\nu,(\alpha)}$ [12]:

$$
\begin{gathered}
H_{\nu,(\alpha)}[g(r)]=\int_{0}^{R} g(r) V_{\nu,(\alpha)}(r, \beta) \sigma(r) d r \equiv \tilde{g}(\beta), \\
H_{\nu,(\alpha)}^{-1}[\tilde{g}(\beta)]=\frac{2}{\pi} \int_{0}^{\infty} \tilde{g}(\beta) V_{\nu,(\alpha)}(r, \beta) \Omega_{v,(\alpha)}(\beta) d \beta \equiv g(r) .
\end{gathered}
$$

Next statement is a mathematical justification of rules (3.20), (3.21). 
Theorem 1. If the function

$$
\begin{gathered}
f(r)=\left[\theta(r) \theta\left(R_{1}-r\right) r^{\alpha_{1}-1 / 2}+\theta\left(r-R_{1}\right) \theta\left(R_{2}-r\right) \cdot 1+\right. \\
\left.+\theta\left(r-R_{2}\right) \theta(R-r) r^{\alpha_{2}+1 / 2}\right] g(r)
\end{gathered}
$$

is continuous, absolutely summable and has bounded variation in the set $(0, R)$, then for any $r \in I_{2}$ integral representation is true

$$
g(r)=\frac{2}{\pi} \int_{0}^{\infty} V_{\nu,(\alpha)}(r, \beta) \int_{0}^{R} g(\rho) V_{\nu,(\alpha)}(\rho, \beta) \sigma(\rho) d \rho \Omega_{\nu,(\alpha)}(\beta) d \beta .
$$

The proof of theorem 1 is performed by the method of delta-shaped sequence (Cauchy kernel or Dirihle kernel) [12].

Let's define the numbers and functions:

$$
\begin{gathered}
d_{1}=a_{1}^{2} \sigma_{1} R_{1}^{2 \alpha_{1}+1} \cdot c_{11,1}^{-1}, d_{2}=a_{2}^{2} \sigma_{2} \cdot c_{11,2}^{-1}, \tilde{g}_{1}(\beta)=\int_{0}^{R_{1}} g_{1}(r) V_{v,(\alpha) ; 1}(r, \beta) \sigma_{1} r^{2 \alpha_{1}-1} d r, \\
\tilde{g}_{2}(\beta)=\int_{R_{1}}^{R_{2}} g_{2}(r) V_{v,(\alpha) ; 2}(r, \beta) \sigma_{2} d r, \tilde{g}_{3}(\beta)=\int_{R_{2}}^{R} g_{3}(r) V_{v,(\alpha) ; 3}(r, \beta) \sigma_{3} r^{2 \alpha_{2}+1} d r, \\
Z_{v,(\alpha) ; i 2}^{k}(\beta)=\left.\left(\tilde{\alpha}_{i 2}^{k} \frac{d}{d r}+\tilde{\beta}_{i 2}^{k}\right) V_{v,(\alpha) ; k+1}(r, \beta)\right|_{r=R_{k}}, \quad i, k=1,2 .
\end{gathered}
$$

Theorem 2. If the function $\left\{B_{\alpha_{1}}^{*}\left[g_{1}(r)\right] ; g_{2}^{\prime \prime}(r) ; B_{v, \alpha_{2}}\left[g_{3}(r)\right]\right\}$ is continuous in the set $I_{2}$, and functions $g_{j}(r)$ satisfy the boundary conditions

$$
\lim _{r \rightarrow 0}\left[r^{2 \alpha_{1}+1}\left(g_{1}^{\prime} V_{v,(\alpha) ; 1}-g_{1} V_{\nu,(\alpha) ; 1}^{\prime}\right)\right]=0,\left.\left(\tilde{\alpha}_{22}^{3} \frac{d}{d r}+\tilde{\beta}_{22}^{3}\right) V_{\nu,(\alpha) ; 3}(r, \beta)\right|_{r=R}=g_{R}
$$

and the conjugate conditions

$$
\left.\left[\left(\tilde{\alpha}_{j 1}^{k} \frac{d}{d r}+\tilde{\beta}_{j 1}^{k}\right) g_{k}(r)-\left(\tilde{\alpha}_{j 2}^{k} \frac{d}{d r}+\tilde{\beta}_{j 2}^{k}\right) g_{k+1}(r)\right]\right|_{r=R_{k}}=\omega_{j k} ; j, k=1,2,
$$

then the basic identity for the integral transform of the HDO $M_{\nu,(\alpha)}$ defined by equality (3.1) is true:

$$
\begin{aligned}
& H_{v,(\alpha)}\left[M_{\nu,(\alpha)}[g(r)]\right]=-\beta^{2} \tilde{g}(\beta)-\sum_{i=1}^{3} k_{i}^{2} \tilde{g}_{i}(\beta)+\left(\tilde{\alpha}_{22}^{3}\right)^{-1} V_{v,(\alpha) ; 3}(R, \beta) \times \\
& \times a_{3}^{2} \sigma_{3} R^{2 \alpha_{2}+1} g_{R}+\sum_{k=1}^{2} d_{k}\left[Z_{v,(\alpha) ; 12}^{k}(\beta) \omega_{2 k}-Z_{v,(\alpha) ; 22}^{k}(\beta) \omega_{1 k}\right] .
\end{aligned}
$$


Proof of Theorem 2. According the rule (3.20) we have that

$$
\begin{gathered}
H_{v,(\alpha)}\left[M_{v,(\alpha)}[g(r)]\right]=\int_{0}^{R} M_{v,(\alpha)}[g(r)] V_{v,(\alpha)}(r, \beta) \sigma(r) d r \equiv \\
\equiv \int_{0}^{R_{1}}\left(a_{1}^{2} B_{\alpha_{1}}^{*}\left[g_{1}(r)\right]\right) V_{v,(\alpha) ; 1}(r, \beta) \sigma_{1} r^{2 \alpha_{1}-1} d r+\int_{R_{1}}^{R_{2}}\left(a_{2}^{2} \frac{d^{2} g_{2}}{d r^{2}}\right) V_{v,(\alpha) ; 2}(r, \beta) \sigma_{2} d r+ \\
+\int_{R_{2}}^{R}\left(a_{3}^{2} B_{v, \alpha_{2}}\left[g_{3}(r)\right]\right) V_{v,(\alpha) ; 3}(r, \beta) \sigma_{3} r^{2 \alpha_{2}+1} d r .
\end{gathered}
$$

Let's integrate by parts twice in (3.26):

$$
\begin{gathered}
H_{v,(\alpha)}\left[M_{\nu,(\alpha)}[g(r)]\right]=\left.a_{1}^{2} \sigma_{1}\left[r^{2 \alpha_{1}+1}\left(\frac{d g_{1}}{d r} V_{v,(\alpha) ; 1}(r, \beta)-g_{1}(r) \frac{d V_{v,(\alpha) ; 1}}{d r}\right)\right]\right|_{R_{0}} ^{R_{1}}+ \\
+\int_{0}^{R_{1}} g_{1}(r)\left(a_{1}^{2} B_{\alpha_{1}}^{*}\left[V_{v,(\alpha) ; 1}(r, \beta)\right]\right) \sigma_{1} r^{2 \alpha_{1}-1} d r+a_{2}^{2} \sigma_{2}\left(\frac{d g_{2}}{d r} V_{v,(\alpha) ; 2}(r, \beta)-\right. \\
\left.-g_{2}(r) \frac{d V_{v,(\alpha) ; 2}}{d r}\right)\left.\right|_{R_{1}} ^{R_{2}}+\int_{R_{1}}^{R_{2}} g_{2}(r)\left(a_{2}^{2} \frac{d^{2} V_{v,(\alpha) ; 2}}{d r^{2}}\right) \sigma_{2} d r+ \\
+\left.a_{3}^{2} \sigma_{3}\left[r^{2 \alpha_{2}+1}\left(\frac{d g_{3}}{d r} V_{v,(\alpha) ; 3}(r, \beta)-g_{3}(r) \frac{d V_{\nu,(\alpha) ; 3}}{d r}\right)\right]\right|_{R_{2}} ^{R}+ \\
+\int_{R_{2}}^{R} g_{3}(r)\left(a_{3}^{2} B_{v, \alpha_{2}}\left[V_{v,(\alpha) ; 3}(r, \beta)\right]\right) \sigma_{3} r^{2 \alpha_{2}+1} d r
\end{gathered}
$$

Due to the boundary condition in the point $r=0$ we obtain that

$$
\lim _{r \rightarrow 0}\left[r^{2 \alpha_{1}+1}\left(\frac{d g_{1}}{d r} V_{\nu,(\alpha) ; 1}(r, \beta)-g_{1}(r) \frac{d V_{\nu,(\alpha) ; 1}}{d r}\right)\right]=0 .
$$

Let's use the basic identity (3.4) for the case if the conjugate conditions are not homogeneous

$$
\begin{aligned}
& {\left.\left[g_{k}^{\prime} V_{\nu,(\alpha) ; k}(r, \beta)-g_{k} V_{v,(\alpha) ; k}^{\prime}(r, \beta)\right]\right|_{r=R_{k}}=\frac{c_{21, k}}{c_{11, k}}\left[g_{k+1}^{\prime}(r) V_{v,(\alpha) ; k+1}-g_{k+1} \times\right.} \\
& \left.\quad \times V_{\nu,(\alpha) ; k+1}^{\prime}\right]\left.\right|_{r=R_{k}}+c_{11, k}^{-1}\left[Z_{v,(\alpha) ; 12}^{k}(\beta) \omega_{2 k}-Z_{v,(\alpha) ; 22}^{k}(\beta) \omega_{1 k}\right] .
\end{aligned}
$$


Due to the basic identity (3.28) in the point $r=R_{1}$ for $\mathrm{k}=1$ we have that

$$
\begin{aligned}
& \left.a_{1}^{2} \sigma_{1} R_{1}^{2 \alpha_{1}+1}\left(g_{1}^{\prime} V_{v,(\alpha) ; 1}-g_{1} V_{v,(\alpha) ; 1}^{\prime}\right)\right|_{R_{1}}-\left.a_{2}^{2} \sigma_{2}\left(g_{2}^{\prime} V_{v,(\alpha) ; 2}-g_{2} V_{v,(\alpha) ; 2}^{\prime}\right)\right|_{R_{1}}= \\
= & \left.\left(a_{1}^{2} \sigma_{1} R_{1}^{2 \alpha_{1}+1} \frac{c_{21,1}}{c_{11,1}}-a_{2}^{2} \sigma_{2}\right)\left(g_{2}^{\prime} V_{v,(\alpha) ; 2}-g_{2} V_{v,(\alpha) ; 2}^{\prime}\right)\right|_{r=R_{1}}+a_{1}^{2} \sigma_{1} R_{1}^{2 \alpha_{1}+1} \cdot c_{11,1}^{-1} \times \\
\times & \left(Z_{v,(\alpha) ; 12}^{1}(\beta) \omega_{21}-Z_{v,(\alpha) ; 22}^{1}(\beta) \omega_{11}\right)=d_{1}\left(Z_{v,(\alpha) ; 12}^{1}(\beta) \omega_{21}-Z_{v,(\alpha) ; 22}^{1}(\beta) \omega_{11}\right),
\end{aligned}
$$

because due to the choice of numbers $\sigma_{1}, \sigma_{2}$ expression

$$
\begin{gathered}
\left(a_{1}^{2} \sigma_{1} R_{1}^{2 \alpha_{1}+1} \frac{c_{21,1}}{c_{11,1}}-a_{2}^{2} \sigma_{2}\right)=\frac{c_{21,1}}{c_{11,1}} R_{1}^{2 \alpha_{1}+1}-\frac{c_{21,1}}{c_{11,1}} R_{1}^{2 \alpha_{1}+1}= \\
=\frac{c_{21,1}}{c_{11,1}} R_{1}^{2 \alpha_{1}+1}(1-1) \equiv 0 .
\end{gathered}
$$

Due to the basic identity (3.28) in the point $r=R_{2}$ for $\mathrm{k}=2$ we have that

$$
\begin{gathered}
\left.a_{2}^{2} \sigma_{2}\left(g_{2}^{\prime} V_{\nu,(\alpha) ; 2}-g_{2} V_{v,(\alpha) ; 2}^{\prime}\right)\right|_{r=R_{2}}-\left.a_{3}^{2} \sigma_{3} R_{2}^{2 \alpha_{2}+1}\left(g_{3}^{\prime} V_{\nu,(\alpha) ; 3}-g_{3} V_{v,(\alpha) ; 3}^{\prime}\right)\right|_{r=R_{2}}= \\
=\left.\left(a_{2}^{2} \sigma_{2} \frac{c_{21,2}}{c_{11,2}}-a_{3}^{2} \sigma_{3} R_{2}^{2 \alpha_{2}+1}\right)\left(g_{3}^{\prime} V_{v,(\alpha) ; 3}-g_{3} V_{\nu,(\alpha) ; 3}^{\prime}\right)\right|_{r=R_{2}}+a_{2}^{2} \sigma_{2} c_{11,2}^{-1} \times
\end{gathered}
$$

$\times\left(Z_{v,(\alpha) ; 12}^{2}(\beta) \omega_{22}-Z_{v,(\alpha) ; 22}^{2}(\beta) \omega_{12}\right)=d_{2}\left(Z_{v,(\alpha) ; 12}^{2}(\beta) \omega_{22}-Z_{v,(\alpha) ; 22}^{2}(\beta) \omega_{12}\right)$, because due to the choice of numbers $\sigma_{2}$ and $\sigma_{3}$ expression

$$
\begin{gathered}
a_{2}^{2} \sigma_{2} \frac{c_{21,2}}{c_{11,2}}-a_{3}^{2} \sigma_{3} R_{2}^{2 \alpha_{2}+1}=\frac{c_{21,1}}{c_{11,1}} \frac{c_{21,2}}{c_{11,2}} R_{1}^{2 \alpha_{1}+1}-\frac{c_{21,1} c_{21,2}}{c_{11,1}} c_{11,2} \frac{R_{1}^{2 \alpha_{1}+1}}{R_{2}^{2 \alpha_{2}+1}} R_{2}^{2 \alpha_{2}+1}= \\
=\frac{c_{21,1} c_{21,2}}{c_{11,1}} c_{11,2} R_{1}^{2 \alpha_{1}+1}(1-1) \equiv 0 .
\end{gathered}
$$

If $\tilde{\alpha}_{22}^{3} \neq 0$, then

$$
\begin{aligned}
& \left.a_{3}^{2} \sigma_{3} R^{2 \alpha_{2}+1}\left(\frac{d g_{3}}{d r} V_{v,(\alpha) ; 3}-g_{3} \frac{d V_{v,(\alpha) ; 3}}{d r}\right)\right|_{r=R}=a_{3}^{2} \sigma_{3}\left(\tilde{\alpha}_{22}^{3}\right)^{-1} R^{2 \alpha_{2}+1} \times \\
& \quad \times\left[\left.\left(\tilde{\alpha}_{22}^{3} \frac{d}{d r}+\tilde{\beta}_{22}^{3}\right) g_{3}\right|_{r=R} \cdot V_{v,(\alpha) ; 3}(R, \beta)-g_{3}(R)\left(\tilde{\alpha}_{22}^{3} \frac{d}{d r}+\tilde{\beta}_{22}^{3}\right) \times\right. \\
& \left.\quad \times\left. V_{v,(\alpha) ; 3}(R, \beta)\right|_{r=R}\right]=\left(\tilde{\alpha}_{22}^{3}\right)^{-1} V_{v,(\alpha) ; 3}(R, \beta) a_{3}^{2} \sigma_{3} R^{2 \alpha_{2}+1} g_{R} .
\end{aligned}
$$

From the differential identities

$$
\begin{gathered}
{\left[a_{1}^{2} B_{\alpha_{1}}^{*}+\left(\beta^{2}+k_{1}^{2}\right)\right] V_{v,(\alpha) ; 1}(r, \beta)=0,\left[a_{2}^{2} F+\left(\beta^{2}+k_{2}^{2}\right)\right] V_{v,(\alpha) ; 2}(r, \beta)=0,} \\
{\left[a_{3}^{2} B_{v, \alpha_{2}}+\left(\beta^{2}+k_{3}^{2}\right)\right] V_{v,(\alpha) ; 3}(r, \beta)=0}
\end{gathered}
$$


we find the functional dependence:

$$
\begin{aligned}
& a_{1}^{2} B_{\alpha_{1}}^{*}\left[V_{\nu,(\alpha) ; 1}(r, \beta)\right]=-\left(\beta^{2}+k_{1}^{2}\right) V_{\nu,(\alpha) ; 1}(r, \beta), a_{2}^{2} \frac{d^{2}}{d r^{2}}\left[V_{v,(\alpha) ; 2}(r, \beta)\right]= \\
= & -\left(\beta^{2}+k_{2}^{2}\right) V_{v,(\alpha) ; 2}(r, \beta), a_{3}^{2} B_{v, \alpha_{2}}\left[V_{\nu,(\alpha) ; 3}(r, \beta)\right]=-\left(\beta^{2}+k_{3}^{2}\right) V_{v,(\alpha) ; 3}(r, \beta) .
\end{aligned}
$$

Let's substitute the obtained dependence (3.29)-(3.32) to the (3.27). We get:

$$
\begin{gathered}
H_{v,(\alpha)}\left[M_{v,(\alpha)}[g(r)]\right]=-\sum_{i=1}^{3}\left(\beta^{2}+k_{i}^{2}\right) \tilde{g}_{i}(\beta)+\sum_{k=1}^{2} d_{k}\left[Z_{v,(\alpha) ; 12}^{k}(\beta) \omega_{2 k}-\right. \\
\left.-Z_{v,(\alpha) ; 22}^{k}(\beta) \omega_{1 k}\right]+\left(\tilde{\alpha}_{22}^{3}\right)^{-1} V_{v,(\alpha) ; 3}(R, \beta) a_{3}^{2} \sigma_{3} R^{2 \alpha_{2}+1} g_{R} .
\end{gathered}
$$

Since the

$$
\sum_{i=1}^{3}\left(\beta^{2}+k_{i}^{2}\right) \tilde{g}_{i}(\beta)=\beta^{2} \sum_{i=1}^{3} \tilde{g}_{i}(\beta)+\sum_{i=1}^{3} k_{i}^{2} \tilde{g}_{i}(\beta)=\beta^{2} \tilde{g}(\beta)+\sum_{i=1}^{3} k_{i}^{2} \tilde{g}_{i}(\beta),
$$

then equality (3.33) coincides with equality (3.25). The theorem is proved.

\section{SOLUTION OF THE PROBLEM}

Let's construct the integral representation of the exact analytical solution of parabolic boundary value problem of conjugation (2.1) - (2.3) by the method of EulerFourier-Bessel hybrid integral transform with spectral parameter.

Let's write the system (2.1) and the zero initial conditions in matrix form:

$$
\begin{gathered}
{\left[\begin{array}{c}
\left(\frac{\partial}{\partial t}+\gamma_{1}^{2}-a_{1}^{2} B_{\alpha_{1}}^{*}\right) u_{1}(t, r) \\
\left(\frac{\partial}{\partial t}+\gamma_{2}^{2}-a_{2}^{2} F\right) u_{2}(t, r) \\
\left(\frac{\partial}{\partial t}+\gamma_{3}^{2}-a_{3}^{2} B_{v, \alpha_{2}}\right) u_{3}(t, r)
\end{array}\right]=\left[\begin{array}{l}
f_{1}(t, r) \\
f_{2}(t, r) \\
f_{3}(t, r)
\end{array}\right],} \\
{\left.\left[\begin{array}{c}
u_{1}(t, r) \\
u_{2}(t, r) \\
u_{3}(t, r)
\end{array}\right]\right|_{t=0}=\left[\begin{array}{l}
0 \\
0 \\
0
\end{array}\right] .}
\end{gathered}
$$

The integral operator $H_{v,(\alpha)}$ is represented as an operator matrix row due to the rule (3.20):

$$
H_{v,(\alpha)}[\cdots]=\left[\int_{0}^{R_{1}} \cdots V_{v,(\alpha) ; 1}(r, \beta) \sigma_{1} r^{2 \alpha_{1}-1} d r \int_{R_{1}}^{R_{2}} \cdots V_{\nu,(\alpha) ; 2}(r, \beta) \sigma_{2} d r\right.
$$




$$
\left.\int_{R_{2}}^{R} \cdots V_{\nu,(\alpha) ; 3}(r, \beta) \sigma_{3} r^{2 \alpha_{2}+1} d r\right] .
$$

Let's apply the operator matrix-row (4.2) to the problem (4.1) according to the matrix multiplication rule. As a result of the main identity (3.25), we get a Cauchy problem [16]:

$$
\begin{gathered}
\left(\frac{d}{d t}+\beta^{2}+\gamma^{2}\right) \tilde{u}(t, \beta) \\
=\tilde{f}(t, \beta)+\sum_{k=1}^{2} d_{k}\left[Z_{v,(\alpha) ; 12}^{k}(\beta) \omega_{2 k}(t)-Z_{v,(\alpha) ; 22}^{k}(\beta) \omega_{1 k}(t)\right]+ \\
+\left(\tilde{\alpha}_{22}^{3}\right)^{-1} V_{v,(\alpha) ; 3}(R, \beta) a_{3}^{2} \sigma_{3} R^{2 \alpha_{2}+1} g_{R}(t) ; \gamma^{2}=\max \left\{\gamma_{1}^{2} ; \gamma_{2}^{2} ; \gamma_{3}^{2}\right\} ;\left.\tilde{u}\right|_{t=0}=0 .
\end{gathered}
$$

Function

$$
\begin{aligned}
\tilde{u}(t, \beta)= & \int_{0}^{t} e^{-\left(\beta^{2}+\gamma^{2}\right)(t-\tau)}\left[\tilde{f}(\tau, \beta)+\sum_{k=1}^{2}\left(Z_{v,(\alpha) ; 12}^{k}(\beta) \omega_{2 k}(\tau)-Z_{v,(\alpha) ; 22}^{k}(\beta) \times\right.\right. \\
& \left.\left.\times \omega_{1 k}(\tau)\right)+\left(\tilde{\alpha}_{22}^{3}\right)^{-1} V_{\nu,(\alpha) ; 3}(R, \beta) a_{3}^{2} \sigma_{3} R^{2 \alpha_{2}+1} g_{R}(\tau)\right] d \tau
\end{aligned}
$$

is the solution of Cauchy problem (4.5).

Integral operator $H_{v,(\alpha)}^{-1}$ due to the rule (3.21), as inverse to (4.2), we represent as the operator matrix-column:

$$
H_{v,(\alpha)}^{-1}[\cdots]=\left[\begin{array}{c}
\frac{2}{\pi} \int_{0}^{\infty} \cdots V_{v,(\alpha) ; 1}(r, \beta) \Omega_{v,(\alpha)}(\beta) d \beta \\
\frac{2}{\pi} \int_{0}^{\infty} \cdots V_{v,(\alpha) ; 2}(r, \beta) \Omega_{v,(\alpha)}(\beta) d \beta \\
\frac{2}{\pi} \int_{0}^{\infty} \cdots V_{v,(\alpha) ; 3}(r, \beta) \Omega_{v,(\alpha)}(\beta) d \beta
\end{array}\right] .
$$

Let's apply operator matrix column (4.7) to matrix element due to matrices multiplication rule, to the matrix-element $[\tilde{u}(t, \beta)]$, where the function $\tilde{u}(t, \beta)$ is defined by formula (4.6). As a result of elementary transformations, we get the integral representation of the only analytical solution of parabolic problem (2.1)-(2.3):

$$
u_{j}(t, r)=\int_{0}^{t} W_{\nu,(\alpha) ; 3 j}(t-\tau, r) g_{R}(\tau) d \tau
$$




$$
\begin{gathered}
+\sum_{k=1}^{2} d_{k} \int_{0}^{t}\left[R_{\nu,(\alpha) ; 12}^{k, j}(t-\tau, r) \omega_{2 k}(\tau)-R_{\nu,(\alpha) ; 22}^{k, j}(t-\tau, r) \omega_{1 k}(\tau)\right] d \tau \\
+\sum_{k=1}^{3} \int_{0}^{t} \int_{R_{k-1}}^{R_{k}} H_{\nu,(\alpha) ; j k}(t-\tau, r, \rho) f(\tau, \rho) \varphi_{k}(\rho) \sigma_{k} d \rho d \tau .
\end{gathered}
$$

Here $R_{0}=0, R_{3}=R, \varphi_{1}(\rho)=\rho^{2 \alpha_{1}-1}, \varphi_{2}(\rho)=1, \varphi_{3}(\rho)=\rho^{2 \alpha_{2}+1}, j=\overline{1,3}$.

In equalities (4) there are principal solutions of parabolic problem (2.1)-(2.3):

1) Green's functions generated by boundary condition at point $r=R$

$$
\begin{gathered}
W_{\nu,(\alpha) ; 3 j}(t, r)=\frac{2}{\pi} \int_{0}^{\infty} e^{-\left(\beta^{2}+\gamma^{2}\right) t}\left(\tilde{\alpha}_{22}^{3}\right)^{-1} V_{\nu,(\alpha) ; 3}(R, \beta) V_{\nu,(\alpha) ; j}(r, \beta) \times \\
\times \Omega_{\nu,(\alpha)}(\beta) d \beta a_{3}^{2} \sigma_{3} R^{2 \alpha_{1}+1},
\end{gathered}
$$

2) Green's functions generated by inhomogeneity of the conjugate conditions

$$
R_{\nu,(\alpha) ; i 2}^{k, j}(t, r)=\frac{2}{\pi} \int_{0}^{\infty} e^{-\left(\beta^{2}+\gamma^{2}\right) t} Z_{v,(\alpha) ; i 2}^{k}(\beta) V_{v,(\alpha) ; j}(r, \beta) \Omega_{v,(\alpha)}(\beta) d \beta ;
$$

$i, k=1,2 ; j=\overline{1,3}$,

3) the influence functions generated by the inhomogeneity of system (2.1)

$$
H_{\nu,(\alpha) ; j k}(t, r, \rho)=\frac{2}{\pi} \int_{0}^{\infty} e^{-\left(\beta^{2}+\gamma^{2}\right) t} V_{\nu,(\alpha) ; j}(r, \beta) V_{\nu,(\alpha) ; k}(\rho, \beta) \Omega_{\nu,(\alpha)}(\beta) d \beta ;
$$

$j, k=\overline{1,3}$.

We get the following theorem as the summary of the above results.

Theorem 3. Let us suppose that the next conditions are true:

1) functions $f_{j}(t, r), g_{R}(t)$ and $\omega_{j k}(t)$ are originals by Laplace;

2) functions $f_{j}(t, r)$ and $g_{j}(r)$ satisfy the conjugate conditions;

3) functions $f(t, r)=\left\{f_{1}(t, r), f_{2}(t, r), f_{3}(t, r)\right\}$ and $g(r)=\left\{g_{1}(r), g_{2}(r), g_{3}(r)\right\}$ are bounded, continuous, absolutely summable with the weight function $\sigma(r)$ and have the bounded variation in the set $I_{2}$;

4) function $F(t, r)=\left\{\frac{\partial}{\partial r} B_{\alpha_{1}}^{*}\left[f_{1}(t, r)\right], \frac{\partial}{\partial r} F\left[f_{2}(t, r)\right], \frac{\partial}{\partial r} B_{v, \alpha_{2}}\left[f_{3}(t, r)\right]\right\}$ is continuously differentiable by $t$ and continuous by $r$ in the set $D_{2}$.

Then in the class of functions $u(t, r)=\left\{u_{1}(t, r), u_{2}(t, r), u_{3}(t, r)\right\}$, which are continuously differentiable by variable $t$ and continuously differentiable by variable $r$ twice in the set $D_{2}$ and satisfy conditions 1), 3), parabolic mixed boundary-value 
problem (2.1)-(2.3) has unique bounded solution, which is determined by the formula (4).

Remark 3. If $\gamma^{2}=\gamma_{1}^{2}>0$, then $k_{1}^{2}=0, k_{2}^{2}=\gamma_{1}^{2}-\gamma_{2}^{2} \geq 0, k_{3}^{2}=\gamma_{1}^{2}-\gamma_{3}^{2} \geq 0$; if $\gamma^{2}=\gamma_{2}^{2}>0$, then $k_{1}^{2}=\gamma_{2}^{2}-\gamma_{1}^{2} \geq 0, k_{2}^{2}=0, k_{3}^{2}=\gamma_{2}^{2}-\gamma_{3}^{2} \geq 0$; if $\gamma^{2}=\gamma_{3}^{2}>0$, then $k_{1}^{2}=\gamma_{3}^{2}-\gamma_{1}^{2} \geq 0, k_{2}^{2}=\gamma_{3}^{2}-\gamma_{2}^{2} \geq 0, k_{3}^{2}=0$.

Remark 4. If initial conditions are not zero, namely

$$
\left.u_{j}(t, r)\right|_{t=0}=g_{j}(r), r \in\left(R_{j-1}, R_{j}\right), j=\overline{1,3}, R_{0}=0, R_{3}=R,
$$

then we go to new function $v(t, r)=\left\{v_{1}(t, r) ; v_{2}(t, r) ; v_{3}(t, r)\right\}$ by formulas

$$
u_{j}(t, r)=v_{j}(t, r)+g_{j}(r), \quad j=\overline{1,3} .
$$

Then it is clear that $\left.v_{j}(t, r)\right|_{t=0}=0, j=\overline{1,3}$.

\section{REFERENCES}

[1] V. Deineka, I. Sergienko, and V. Skopetsky, Models and methods of solving of problems with conjugate conditions. Kyiv: Naukova Dumka, 1998.

[2] V. Gorodetsky, The boundary properties of smooth solutions in the layer of parabolic equations. Chernivtsi: Ruta, 1998.

[3] A. Gromyk, I. Konet, and M. Leniuk, The temperature fields in the piece-homogeneous spatial environments. Kamenets-Podilsky: Abetka-Svit, 2011.

[4] I. Konet and M. Leniuk, The temperature fields in the flat piece-homogeneous orthotropic domains. Kyiv: Institute of Mathematics of NAS of Ukraine, 1997.

[5] I. Konet and M. Leniuk, Stationary and non-stationary temperature fields in cylindric-circle domaines. Chernivtsi: Prut, 2001.

[6] I. Konet and M. Leniuk, Integral transforms of Mehler-Fock type. Chernivtsi: Prut, 2002.

[7] I. Konet and M. Leniuk, The temperature fields in the piece-homogeneous cylindrical domains. Chernivtsi: Prut, 2004.

[8] A. Kurosh, Higher algebra course. Moscow: Nauka, 1971.

[9] M. Leniuk, Integral transforms generated by Euler differential operator of the second order. Chernivtsi: Prut, 2012.

[10] M. Leniuk and H. Mikhalevska, Integral transforms of Kontorovich-Lebedev type. Chernivtsi: Prut, 2002.

[11] M. Leniuk and M. Shynkaryk, Hybrid integral (Fourier, Bessel, Legendre) transforms. Part 1. Ternopil: Ekon. dumka, 2004.

[12] M. Leniuk and M. Shynkaryk, Hybrid integral (Fourier, Euler, Bessel, Legendre) transforms. Part 2. Ternopil: Ekon. dumka, 2011.

[13] M. Matiychuk, Parabolic and elliptic boundary value problems with the features. Chernivtsi: Prut, 2003.

[14] I. Sergienko, V. Skopetsky, and V. Deineka, Mathematic modeling and the study of processes in heterogeneous environments. Kyiv: Naukova Dumka, 1991.

[15] L. Shvarts, Mathematical Methods for Physical Sciences. Moscow: Mir, 1965.

[16] V. Stepanov, The course of differential equations. Moscow: Fizmatgiz, 1959.

[17] A. Tikhonov and A. Samarsky, The equations of mathematical physics. Moscow: Nauka, 1972. 
Authors' addresses

\section{Konet}

Ivan Ohienko Kamianets-Podilsky National University, Physics and Mathematics faculty, 61 Ohienka St., 32300 Kamianets-Podilsky, Ukraine

E-mail address: konet510ukr.net

\section{T. Pylypiuk}

Ivan Ohienko Kamianets-Podilsky National University, Physics and Mathematics faculty, 61 Ohienka St., 32300 Kamianets-Podilsky, Ukraine

E-mail address: t-myh@i.ua 